\title{
Studies on the Biosynthesis of Biotin
}

\section{PRODUCTION OF BIOTIN AND BIOTIN-LIKE COMPOUNDS BY A PSEUDOMONAD}

\author{
By A. H. ROSE, MAHBOOB ILAHI AND MARIANNE V. KELEMEN* \\ Department of Microbiology, University of Newcastle upon Tyne
}

(Received 11 November 1964)

\begin{abstract}
1. Filtrates from cultures of a strain of Pseudomonas aeruginosa, grown in a basal glucose-ammonium chloride-vitamins-salts medium, possessed biotin activity as detected by microbiological assays. Exponential-phase culture filtrates contained biotin and desthiobiotin in the approximate ratio $1: 3$, with smaller amounts of biotin sulphoxide and three unidentified compounds with biotin activity. 2. The addition of malonate, adipate or pimelate to the basal medium stimulated the production of compounds with biotin activity; this effect was enhanced when these compounds were included in the medium as the major carbon source. Succinate, glutarate, suberate, fumarate or oxaloacetate did not stimulate the production of compounds with biotin activity. The ratio of biotin to desthiobiotin in filtrates from cultures grown in medium containing malonate as the carbon source was about $1: 1$. Experiments in which mixtures of malonate and pimelate were included in the medium as the carbon sources showed that these acids probably make a similar contribution in biotin biosynthesis. 3. A number of heterocyclic compounds, including several containing the ureido group (-NH-CO-NH-), were included in the basal medium but none of them stimulated the production of compounds with biotin activity to any marked degree. 4. Several amino acids, particularly cysteine (or cystine) and lysine, when added individually as supplements to the basal medium, stimulated the production of compounds with biotin activity. Filtrates from cultures grown in medium supplemented with cysteine contained approximately equal proportions of biotin and desthiobiotin. A much greater stimulation in the production of compounds with biotin activity was obtained when certain amino acids were included in the medium as the major source of nitrogen or carbon and nitrogen; ornithine, citrulline and argininosuccinate had the most marked effect. The ratio of biotin to desthiobiotin in filtrates from these cultures was usually greater than in filtrates from cultures grown in basal medium. 5-Aminovalerate also caused some stimulation when used as the nitrogen source, but urea was inactive. The effect of binary mixtures of certain amino acids was also examined. 5. The results are discussed in relation to the possible role of the stimulatory compounds during biotin biosynthesis.
\end{abstract}

Although work reported during recent years has led to a more complete understanding of the metabolic role of biotin, comparatively little is known about the biosynthesis of this B-group vitamin (for a review see Goodwin, 1963). Living cells usually synthesize extremely small amounts of biotin and compounds with biotin-like activity, and this largely explains why so little is known about the biosynthesis of these compounds. It has been suggested that pimelate is concerned in biotin biosynthesis since it replaces biotin as a growth factor for a strain of Corynebacterium diphtheriae (du Vigneaud,

* Present address: Department of Biochemistry, Royal Free Hospital School of Medicine, London, W.C.1.
Dittmer, Hague \& Long, 1942a) and in certain thermophilic bacteria (Campbell \& Williams, 1953) and stimulates the production of biotin by Asper. gillus niger (Eakin \& Eakin, 1942). Eisenberg (1962) and Elford \& Wright (1962) have reported evidence for the incorporation of radioactive pimelate into the biotin molecule. The stimulatory effect of pimelate on biotin production by $A$. niger is enhanced in the presence of cysteine, which suggests that this amino acid may contribute carbon, nitrogen and sulphur atoms during the biosynthesis of biotin (Eakin \& Eakin, 1942). Desthiobiotin has been proposed as an intermediate in biotin biosynthesis since it can substitute for the vitamin with many biotin-requiring 
micro-organisms (Leonian \& Lilly, 1945; Lichstein, 1955) and is accumulated by a biotin-requiring mutant of Penicillium chrysogenum (Tatum, 1945).

The only report that has attempted to explain the biosynthetic origin of all of the atoms in the biotin molecule is by Lezius, Ringelmann \& Lynen (1963). Based solely on the nature of the products obtained by the controlled degradation of biotin isolated from an achromobacter grown in a medium containing isovaleric acid with $[3-14 \mathrm{C}]$ cysteine or [ $\left.{ }^{14} \mathrm{C}\right]$ bicarbonate, these workers put forward a putative biosynthetic scheme in which the atoms in the biotin molecule are contributed by carbamoyl phosphate, cysteine and pimelyl-CoA.

The experiments reported in the present paper were carried out as part of a study of the biosynthesis of biotin and were undertaken to evaluate the stimulatory effect of various compounds on the production of biotin and biotin-like compounds by a pseudomonad and to ascertain where possible the contributions which these stimulatory compounds might make during biotin biosynthesis by the bacterium. Certain of the data included in this paper have already been reported (Rose \& Mahboob Ilahi, 1963).

\section{METHODS}

Organism. The bacterium used in this study was a strain of Pseudomonas aeruginosa (no. 2447) isolated in the Department of Bacteriology, Royal Victoria Infirmary, Newcastle upon Tyne. This organism was selected after preliminary experiments in which a number of bacteria were examined for the ability to produce appreciable amounts of extracellular biotin. The bacterium was maintained on nutrient-agar slopes, and a freshly grown slope was used for preparing the inoculum in each experiment. The bacteria were grown in a medium containing (per 1 . of water): glucose, 20g.; $\mathrm{Na}_{2} \mathrm{HPO}_{4}, 6.0$ g.; $\mathrm{KH}_{2} \mathrm{PO}_{4}, 3.0 \mathrm{~g}$; $\mathrm{NH}_{4} \mathrm{Cl}, 2 \cdot 0$ g.; $\mathrm{NaCl}, 3 \cdot 0 \mathrm{~g}$.; $\mathrm{MgSO}_{4}, 7 \mathrm{H}_{2} \mathrm{O}, 100 \mathrm{mg}$.; nicotinic acid, $0.4 \mathrm{mg}$; thiamine hydrochloride, $1.0 \mathrm{mg}$.; calcium D-pantothenate, $1.0 \mathrm{mg}$.; pyridoxine hydrochloride, $1.0 \mathrm{mg}$.; riboflavine, $0.25 \mathrm{mg}$. Unless otherwise stated, the pH of the medium was $7 \cdot 5$. This is referred to below as 'basal medium'. In certain experiments other compounds were included in the medium as supplements, as the major carbon source (in place of glucose), as the major nitrogen source (replacing $\mathrm{NH}_{4} \mathrm{Cl}$ ) or as the major carbon and nitrogen source (replacing glucose and $\left.\mathrm{NH}_{4} \mathrm{Cl}\right)$. Portions $(40 \mathrm{ml}$.) of glucose-free medium were dispensed in $350 \mathrm{ml}$. conical flasks that were plugged with cotton wool and sterilized by autoclaving at $120^{\circ}$ for $10 \mathrm{~min}$. After cooling, $10 \mathrm{ml}$. of a sterile $10 \%(\mathrm{w} / \mathrm{v})$ solution of glucose was added aseptically to each flask of medium. In experiments designed to study the effect of argininosuccinate on the production of compounds with biotin activity, portions ( $4 \mathrm{ml}$.) of double-strength glucosefree medium, with or without $\mathrm{NH}_{4} \mathrm{Cl}$, were dispensed into $50 \mathrm{ml}$. conical flasks that were plugged and sterilized as described above. After cooling, the medium was supplemented aseptically with $2 \mathrm{ml}$. of the sterile glucose solution or with a solution of barium argininosuccinate of the appropriate concentration, or both, and made up to $10 \mathrm{ml}$. with sterile water. Solutions of barium argininosuccinate were sterilized by filtration through a sintered-glass filter (Baird and Tatlock K 702; porosity 5).

The inoculum was prepared by suspending bacteria from a freshly grown agar slope in $6 \mathrm{ml}$. of $0.85 \% \mathrm{NaCl}$ to give a density of $0.08-0.12 \mathrm{mg}$. dry wt. of bacteria $/ \mathrm{ml}$. The suspension was centrifuged at $1500 \mathrm{~g}$ at room temperature and the supernatant discarded. The bacteria were then washed three times with further portions $(6 \mathrm{ml}$.) of sterile $0.85 \%$ $\mathrm{NaCl}$ and resuspended in $6 \mathrm{ml}$. of $0.85 \% \mathrm{NaCl}$. A portion $(0.4 \mathrm{ml}$.) of this suspension was added to each flask of medium (50 ml.) and proportionately less to $10 \mathrm{ml}$. portions of medium. The cultures were incubated in a New Brunswick Gyrotary shaker-incubator (model G 25) and, unless otherwise specified, were at a temperature of $37^{\circ}$ and shaken at 170 rotations/min. These conditions were most favourable for production in basal medium. Growth was measured turbidimetrically in Samco tubes (Northam \& Norris, 1951) in the Hilger Spekker absorptiometer (model H 760) with neutral green-grey $\mathbf{H} 508$ filters and a water blank. Turbidity readings were related to dry wt. of bacteria. Culture filtrates were obtained by centrifuging portions of culture at $145000 \mathrm{~g}$ for $20 \mathrm{~min}$. at $0 \pm 1^{\circ}$ in the MSE Superspeed 40 ultracentrifuge (rotor no. 309 or 402). Filtrates were either used immediately or stored at $-20^{\circ}$ until required.

Assays of biotin activity. Total biotin activity in culture filtrates and in extracts of the pseudomonad was determined microbiologically with Lactobacillus plantarum (L.arabinosus 17/5; NCIB8126), as described by Barton-Wright (1952). The biotin activity in culture filtrates was shown to be due to several compounds with biotin activity in addition to biotin. Culture filtrates were adjusted to $\mathrm{pH6} 8$, and diluted 10-200-fold before assay, which was carried out in $100 \mathrm{~mm} . \times 12 \mathrm{~mm}$. tubes covered with aluminium caps (Oxoid; Oxo Ltd., London, E.C.4) and containing $3 \mathrm{ml}$. of medium. After incubation for $72 \mathrm{hr}$. at $30^{\circ}$ the cultures were titrated against $0.05 \mathrm{~N}-\mathrm{NaOH}$, with bromothymol blue $[0.04 \%(w / v)$ in $50 \%(w / v)$ ethanol] as an internal indicator (pH6.8). A standard dose-response curve was prepared with each assay. The media used for growing the pseudomonad contained no detectable biotin activity. It was also shown that none of the compounds added to the basal medium at the concentrations used affected the response of the lactobacillus to biotin.

The contents of free and combined biotin in the pseudomonad were determined as described by Barton-Wright (1952).

Chromatography. Biotin and biotin-like compounds in culture filtrates were separated by descending one-dimensional chromatography on Whatman no. 1 paper with each of the following solvent systems: I, butan-1-ol-acetic acidwater $(4: 1: 5$, by vol.); II, butan-1-ol-water $(43: 7, v / v)$. Papers were irrigated at room temperature $\left(20-22^{\circ}\right)$ and, after the solvent front had travelled $28-30 \mathrm{~cm}$., the chromatogram was removed and dried. Strips, $2 \mathrm{~cm}$. wide and running the length of each track, were removed from the chromatogram and cut into numbered $1 \mathrm{~cm}$. squares. The total biotin activity on each square was determined with a biotin-requiring strain of Saccharomyces cerevisiae Fleischmann (Rose, 1960). The assay medium used was that described by Rose \& Nickerson (1956) supplemented with vitamin-free casein hydrolysate (Difco; l g./1.) (Hertz, 1943). Each square of paper was added to a separate portion $(6 \mathrm{ml}$.) of yeast assay medium in Samco tubes and 
sterilized by autoclaving momentarily at $110^{\circ}$. The medium was inoculated as described by Rose (1960). Cultures were incubated statically at $25^{\circ}$ for $30-36 \mathrm{hr}$. and, after the squares of paper had been removed with forceps, the turbidity of the culture was measured. Because of the difference in the response of the yeast to biotin and biotin sulphoxide (Melville, Genghof \& Lee, 1954), and possibly also to the unidentified biotin-like compounds in the pseudomonad culture filtrates, the total biotin activity of the squares is given as the turbidity reading of the yeast culture. With this assay organism, there is a linear relationship between turbidity $(0 \cdot 20-0 \cdot 80)$ in Samco tubes and biotin concentration (approx. 0.02-0.15m $\mu$ g. of biotin/ml.). Chromatograms of a standard biotin solution were included in each experiment. In certain experiments, sterile portions of assay medium were supplemented before inoculation with a solution of avidin that had been sterilized by filtration through a sintered-glass filter (Baird and Tatlock K 702; porosity 5).

Chemicals. D-Biotin was obtained from General Biochemicals Inc., Chagrin Falls, Ohio, U.S.A., and DL-desthiobiotin from L. Light and Co. Ltd., Colnbrook, Bucks. Barium argininosuccinate was obtained from the Sigma Chemical Co., St Louis, Mo., U.S.A., and avidin from Nutritional Biochemicals Inc., Cleveland, Ohio, U.S.A. A sample of biocytin (batch L511199-0-2) was kindly provided by Dr W. B. Woodruff of Merck, Sharp and Dohme, Rahway, N.J., U.S.A. All other chemicals used in this study were of the highest purity available commercially.

Biotin D-sulphoxide was prepared from D-biotin by the method of Wright, Cresson, Valiant, Wolf \& Folkers (1954), and biotin $D$-sulphone according to the technique described by Hofmann, Melville \& du Vigneaud (1941). The purity of each derivative was checked by melting-point determinations and by chromatography.

\section{RESULTS} Production of biotin and biotin-like compounds in
basal medium

Variations in incubation temperature $\left(25-40^{\circ}\right)$, initial $\mathrm{pH}$ of the medium (pH 6.0-8.0) and shaking speed (148-268 rotations/min.) had little or no effect on the rate of growth of the bacterium, although they affected the duration of the lag phase of growth. Under most of the conditions examined, the biotin activity of the filtrate was greatest during the mid-exponential phase of growth and thereafter declined, presumably because of the breakdown of biotin and possibly also of biotin-like compounds by the pseudomonad (Fig. 1).

The total biotin activity in filtrates from cultures of the bacterium grown in basal medium and harvested in the mid-exponential phase of growth was about $0.25 \mathrm{~m} \mu \mathrm{g}$. of biotin equivalent $/ \mathrm{ml}$. In bacteria harvested from these cultures, the total amount of compounds with biotin activity that were bound to cell constituents $(2.04 \mathrm{~m} \mu \mathrm{g}$. of biotin equivalent $/ \mathrm{mg}$. dry wt.) was approximately five times as great as the content of water-soluble compounds with biotin activity. This distribution of biotin in the pseudo-

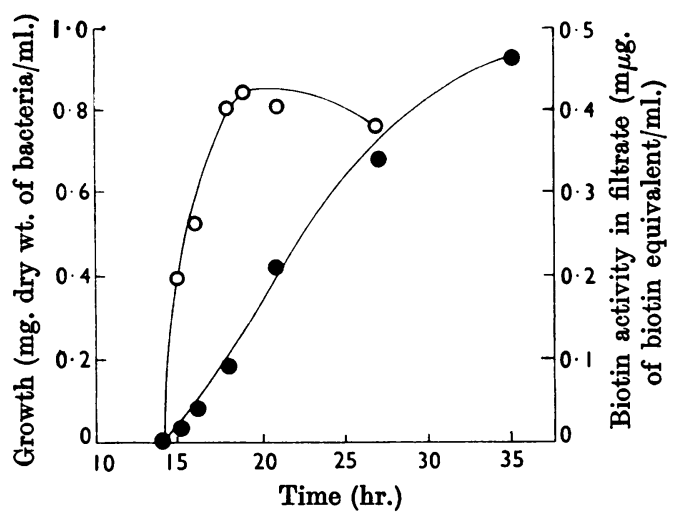

Fig. 1. Effect of incubation time on growth of $(\bullet)$, and production of compounds with biotin activity by $(0)$, the pseudomonad in basal medium (pH7.5). Cultures (50 ml.) were incubated at $37^{\circ}$ and shaken at 170 rotations/min.

monad and the culture filtrate was similar to that reported by Landy \& Dicken (1941) for other bacteria.

The results of chromatography of filtrates from cultures grown in basal medium are shown in Figs. $2(a)$ and $2(b)$; the yeast was used as the assay organism in these experiments. By using solvent system I, the filtrates were shown to contain mainly biotin or desthiobiotin or both $\left(R_{F} 0 \cdot 85\right)$, together with smaller amounts of biotin sulphoxide $\left(R_{F} 0.59\right)$ and three unidentified compounds with biotin activity with $R_{F}$ values $0.07,0.18$ and 0.33 . Neither biocytin $\left(R_{F} 0 \cdot 69\right)$ nor biotin sulphone $\left(R_{F} 0 \cdot 89\right)$ could be detected on these chromatograms. With solvent system I we were unable to separate biotin and desthiobiotin, although Wright \& Driscoll (1954) claimed that they could. Biotin $\left(R_{F} 0 \cdot 33\right)$ and desthiobiotin $\left(R_{F} 0 \cdot 79\right)$ could, however, be separated in solvent system II, and it was shown (Fig. 2b) that the filtrates from mid-exponential phase cultures of the pseudomonad contained biotin and desthiobiotin in the ratio approx. 1:3 (the dose-response curves of the yeast for D-biotin and D-desthiobiotin are almost identical). Biotin sulphoxide $\left(R_{F} \mathbf{0 . 5 5}\right)$ was also detected on chromatograms of filtrates run in solvent system II. Two other peaks $\left(R_{F} 0 \cdot 14\right.$ and 0.66) were detected on chromatograms of filtrates run in solvent system II, so that it is possible that one of the three unidentified biotin-like compounds present in the filtrate migrated in this solvent to a position near that of biotin or desthiobiotin. But, since there were only small amounts of these unidentified compounds present, as judged by their ability to replace the biotin requirement of the yeast, this should not have appreciably affected the calculation of the relative proportions of biotin and desthiobiotin in the filtrates. 

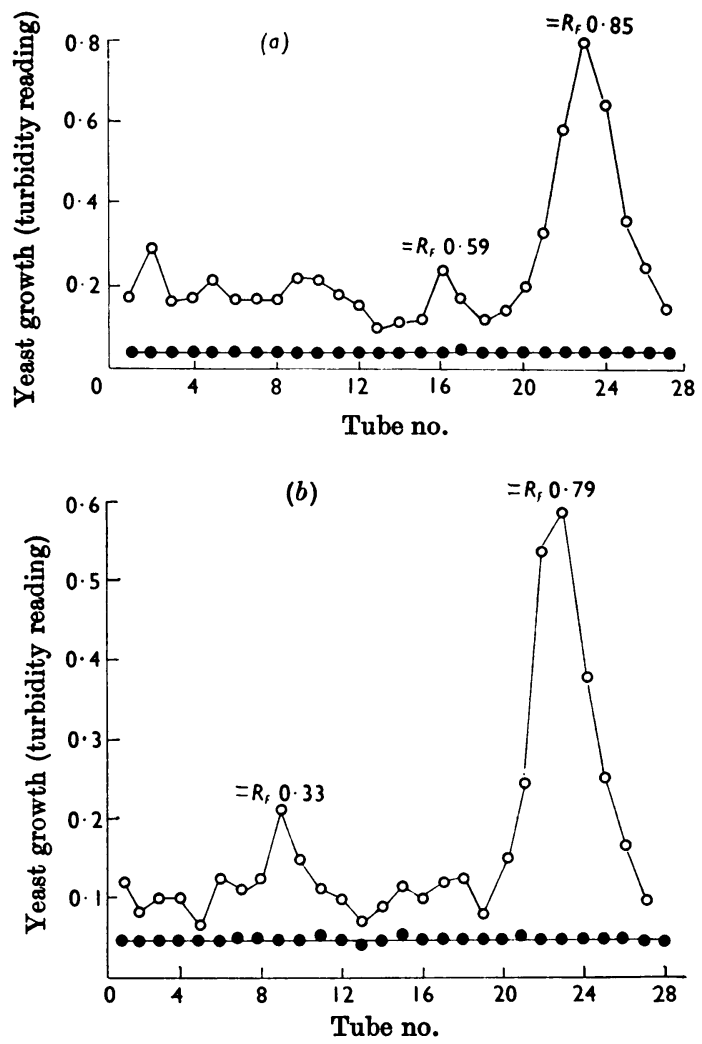

Fig. 2. Separation of compounds with biotin activity by paper chromatography. Bacterial cultures in basal medium were harvested when growth reached $0.25 \mathrm{mg}$. dry wt. of bacteria $/ \mathrm{ml}$. Portions (about $0 \cdot 10 \mathrm{ml}$.) of supernatant, with an activity equivalent to $0.40 \mathrm{~m} \mu \mathrm{g}$. of biotin $/ \mathrm{ml}$., were applied to the chromatogram paper which was then treated as described in the Methods section with solvent system I $(a)$ or II (b). O, Growth of the yeast assay organism on samples from untreated cultures; $\bullet$, growth of the yeast on samples from cultures supplemented with $0 \cdot 10 \mathrm{ml}$. of a sterile solution of avidin ( $164 \mu \mathrm{g}$. of protein $/ \mathrm{ml}$.). Chromatography of known compounds showed that the filtrates contained biotin, desthiobiotin and biotin sulphoxide. In (a), the peak at $R_{F} 0.59$ is biotin sulphoxide, and that at $R_{F} 0.85$ is biotin + desthiobiotin; in (b) the peak at $R_{F} 0.33$ is biotin, and that at $R_{F} 0.79$ is desthiobiotin. In solvent system I, three unidentified compounds with biotin activity $\left(R_{F} 0 \cdot 07,0 \cdot 18\right.$ and 0.33$)$ were detected.

When the biotin activity on the chromatograms run in solvent system I was assayed with the lactobacillus, the height of the peak at $R_{F} 0 \cdot 85$ was smaller than on chromatograms assayed with the yeast. This was because desthiobiotin cannot replace biotin as a growth factor with the lactobacillus. The unidentified compound with $R_{F} 0.07$ in solvent system I was also unable to satisfy the biotin requirement of the lactobacillus.
Avidin, a glycoprotein obtained from egg white, combines with biotin and certain biotin-like compounds to give biologically inactive complexes (Eakin, Snell \& Williams, 1941). Although little is known of the mechanism of the biotin-binding action of avidin, it is thought that combination occurs only with compounds containing a ureido group (du Vigneaud, Dittmer, Hofmann \& Melville, 1942b; Green, 1963). The effects of adding an excess of avidin to the yeast assay cultures are also shown in Figs. 2(a) and 2(b). The inability of the three unidentified compounds to replace the biotin requirement of the yeast in avidin-supplemented cultures indicated that they contained the ureido group.

\section{Effect of individual dicarboxylic acids, heterocyclic compounds and amino acids on the production of compounds with biotin activity}

Dicarboxylic acids. Several reports have appeared showing that saturated aliphatic dicarboxylic acids stimulate biotin production by micro-organisms (du Vigneaud et al. 1942a; Eisenberg, 1962; Elford \& Wright, 1962). However, there was no stimulation in the production by the pseudomonad of compounds with biotin activity when short-chain saturated aliphatic dicarboxylic acids $\left(\mathrm{C}_{3}-\mathrm{C}_{8}\right)$ were added individually to basal medium. But, when these acids were included in the medium as the major carbon source $(50 \mathrm{~mm})$ in place of glucose, malonate and, to a smaller extent, adipate and pimelate caused a stimulation in production as compared with that in basal medium (Table 1). In medium containing malonate or succinate as the

Table 1. Production of compounds with biotin activity by the pseudomonad in media containing individual saturated aliphatic dicarboxylic acids as the major carbon source

The acids were present at $50 \mathrm{~mm}$. Cultures $(50 \mathrm{ml}$.) were removed when growth reached the values indicated and the total biotin activity in the culture filtrates was determined by the lactobacillus assay.

Biotin activity in filtrate ( $\mathrm{m} \mu \mathrm{g}$. of biotin equivalent/ml.)

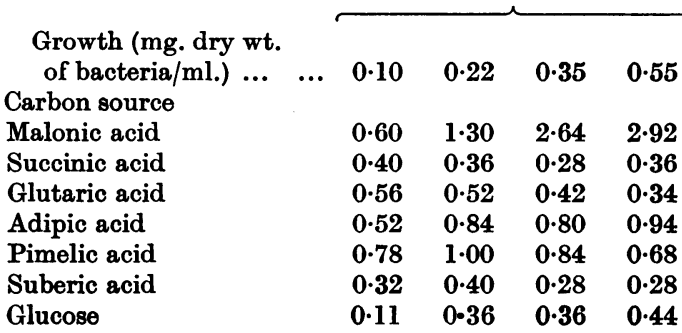


carbon source, the bacterium grew as rapidly as in basal medium, but when acids of longer chain length were used the duration of the lag phase of growth was increased.

Chromatography of filtrates from cultures containing malonate $(50 \mathrm{~mm})$ as the major carbon source showed that the unidentified compound $\left(R_{F} 0.33\right.$ in solvent I) was not present in these filtrates and that the ratio of biotin to desthiobiotin in the filtrates approached 1:1.

Heterocyclic compounds. The biotin molecule can beconsidered as being constructed from a substituted imidazole ring and it is conceivable that, in the biosynthesis of the vitamin, an intact imidazole ring is contributed by purines, pyrimidines or related compounds. Anumber of heterocyclic compoundsincluding several containing the ureido (-NH-CO-NH-) group were examined for the ability to stimulate the production by the pseudomonad of compounds with biotin activity but, with the exception of cytosine and thymine, each of which brought about a small increase in production, none was found to be active. Certain of these compounds, particularly adenine, guanine and uric acid, caused a decrease in production.

Amino acids. The presence of nitrogen atoms in the biotin molecule suggested that amino acids might be required in the biosynthesis of the vitamin. The effect of adding individual amino acids to the basal medium is shown in Table 2. Most amino acids caused an increase of up to $100 \%$ in production as compared with that in amino acidfree medium, suggesting some non-specific effect on vitaminbiosynthesis. However, cysteine(orcystine), lysine and, to a smaller extent, leucine, isoleucine, norleucine, asparagine and glutamine caused a somewhat greater stimulation. Chromatography with solvent system I showed that filtrates from cultures of the bacterium grown in basal medium supplemented with cysteine contained a slightly greater proportion of biotin sulphoxide as compared with filtrates from cultures grown in unsupplemented medium. With solvent system II, it was shown that the ratio of biotin to desthiobiotin was lower $(1: 1)$ in these filtrates as compared with filtrates from cultures of the pseudomonad grown in cysteine-free medium.

Biotin activity in the filtrates was very much greater when certain amino acids, particularly citrulline, ornithine or, to a smaller extent, lysine or aspartate, were included in the medium either as the major nitrogen source (at $5 \mathrm{~mm}$ ) or as the major source of carbon and nitrogen (at $50 \mathrm{~mm}$ ) (Table 3). Growth of the pseudomonad in certain of these media, particularly those containing citrulline or lysine as the major carbon and nitrogen source, was much slower than in basal medium. The bacteria were unable to grow in media containing either
Table 2. Production of compounds with biotin activity by the pseudomonad in basal media supplemented with individual amino acids

Amino acids were incorporated into basal medium at the concentrations stated. Cultures $(50 \mathrm{ml}$.) were removed when growth reached $0.25 \mathrm{mg}$. dry wt. of bacteria $/ \mathrm{ml}$. and the total biotin activity in the culture filtrate was determined by the lactobacillus assay.

Biotin activity in filtrate (m $\mu$ g. of biotin equivalent/mg. dry wt. of bacteria)

\begin{tabular}{|c|c|c|c|c|}
\hline $\begin{array}{l}\text { Concn. of amino } \\
\text { acid in medium } \\
(\mathrm{mg} / \mathrm{ml} .) \ldots \quad \ldots\end{array}$ & 0.0 & $0 \cdot 10$ & $1 \cdot 0$ & $5 \cdot 0$ \\
\hline \multicolumn{5}{|l|}{ Amino acid } \\
\hline DL-Alanine & $0 \cdot 80$ & $1 \cdot 76$ & 0.96 & 0.96 \\
\hline L-Arginine & $0 \cdot 81$ & $1 \cdot 88$ & $1 \cdot 68$ & $1 \cdot 08$ \\
\hline L-Asparagine & $0 \cdot 80$ & $2 \cdot 16$ & $1 \cdot 76$ & $1 \cdot 84$ \\
\hline L-Aspartic acid & 0.96 & $1 \cdot 68$ & 0.96 & $1 \cdot 86$ \\
\hline DL-Citrulline & $0 \cdot 80$ & $1 \cdot 60$ & $1 \cdot 28$ & 1.86 \\
\hline L-Cysteine & 0.96 & $1 \cdot 68$ & $2 \cdot 40$ & insol. \\
\hline L-Cystine & 0.96 & $1 \cdot 44$ & $2 \cdot 72$ & insol. \\
\hline L-Glutamic acid & $1 \cdot 12$ & $1 \cdot 28$ & $1 \cdot 76$ & 1.56 \\
\hline L-Glutamine* & 0.96 & $1 \cdot 04$ & $1 \cdot 44$ & $2 \cdot 24$ \\
\hline Glycine & 0.96 & $1 \cdot 68$ & 0.88 & 0.72 \\
\hline L-Histidine & 0.81 & $1 \cdot 40$ & $1 \cdot 24$ & 0.84 \\
\hline DL-Isoleucine & $1 \cdot 20$ & $1 \cdot 84$ & $2 \cdot 08$ & $2 \cdot 16$ \\
\hline L-Leucine & 0.88 & $2 \cdot 24$ & 1.92 & $1 \cdot 28$ \\
\hline DL-Norleucine & 0.88 & $2 \cdot 40$ & $2 \cdot 24$ & $2 \cdot 08$ \\
\hline L-Lysine & 0.80 & $0 \cdot 88$ & 0.92 & $3 \cdot 84$ \\
\hline L-Methionine & 0.86 & $1 \cdot 16$ & 1.08 & 0.80 \\
\hline L-Ornithine & 0.80 & 0.48 & 0.72 & $1 \cdot 60$ \\
\hline L-Proline & 1.04 & $1 \cdot 04$ & $1 \cdot 12$ & 0.80 \\
\hline L-Serine & 1.20 & 1.84 & $1 \cdot 28$ & $1 \cdot 28$ \\
\hline L-Threonine & 0.80 & $1 \cdot 20$ & $1 \cdot 76$ & 0.48 \\
\hline L-Tryptophan & 1.04 & $0 \cdot 80$ & $1 \cdot 20$ & $1 \cdot 74$ \\
\hline L-Tyrosine & $1 \cdot 16$ & $1 \cdot 12$ & 0.80 & insol. \\
\hline L-Valine & 1.04 & 1.04 & 0.80 & 1.04 \\
\hline
\end{tabular}

* Glutamine was sterilized separately by Seitz filtration and added aseptically to the basal medium. The change in glutamine concentration caused by Seitz filtration of the solution was negligible. Glutamine was determined by the method of Smith \& Agiza (1957).

cysteine or cystine as the major source of nitrogen or carbon and nitrogen. Biotin activity was greatest in filtrates from cultures grown in medium containing ornithine as the carbon and nitrogen source, the activity in these filtrates being more than 40 times as great as that in filtrates from cultures grown in basal medium. In media containing individual amino acids as the major source of nitrogen the production of compounds with biotin activity was greatest with citrulline.

Chromatographic examination of filtrates from cultures of the bacterium grown in medium containing ornithine or lysine as the nitrogen or carbon and nitrogen source gave similar results in solvent 
Table 3. Production of compounds with biotin activity by the pseudomonad in media containing individual amino acids, or products of metabolism of these amino acids, as sources of nitrogen or carbon and nitrogen

Compounds were included in the medium as the major source of nitrogen $(\mathrm{N} ; 5 \mathrm{~mm})$ or as the major source of carbon and nitrogen $(\mathrm{C}+\mathrm{N} ; 50 \mathrm{mM})$. Cultures $(50 \mathrm{ml}$.) were removed when growth reached the values indicated and the biotin activity in the culture filtrates was determined by the lactobacillus assay.

Biotin activity in filtrate ( $\mathrm{m} \mu \mathrm{g}$. of biotin equivalent/ml.)

$\quad$ Compound
5-Aminovalerate
L-Arginine
L-Asparagine
L-Aspartate
DL-Citrulline
L-Glutamate
L-Lysine
L-Ornithine
Urea
Basal medium

Growth (mg. dry wt. of

bacteria/ml.) $\quad \ldots \quad \ldots \quad 0 \cdot 10$

$\begin{array}{cr}\mathrm{N}^{2} & \mathrm{C}+\mathrm{N} \\ 0.80 & 0.28 \\ 0.46 & 0.92 \\ 0.22 & 0.70 \\ 1.08 & 0.80 \\ 6.80 & 3.80 \\ 0.36 & 1.08 \\ 1.34 & 1.68 \\ 1.30 & 15.60 \\ 0.08 & -\end{array}$

$\begin{array}{cr}\mathrm{Cr}_{\mathrm{N}} & \mathrm{C}+\mathrm{N} \\ 0.70 & 0.65 \\ 0.44 & 0.24 \\ 0.52 & 1.48 \\ 1.20 & 0.84 \\ 8.40 & 4.00 \\ 0.40 & 0.72 \\ 2.64 & 1.56 \\ 2.40 & 16.00 \\ 0.30 & -\end{array}$

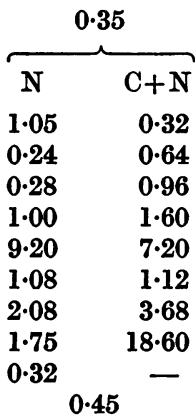

\begin{tabular}{rr}
\multicolumn{1}{c}{$\mathbf{N}$} & $\mathbf{C}+\mathrm{N}$ \\
1.40 & 0.25 \\
0.28 & 0.80 \\
0.16 & 0.42 \\
0.84 & 3.44 \\
11.20 & 12.00 \\
1.04 & 1.08 \\
1.56 & 4.00 \\
1.35 & 16.80 \\
0.32 & - \\
\multicolumn{2}{c}{0.41}
\end{tabular}

system I to those obtained with filtrates from cultures grown in basal medium. But, in medium containing citrulline as the carbon and nitrogen source, there was an increased production of one of the unidentified biotin-like compounds $\left(R_{F} 0.33\right.$ in solvent system I). The ratios of biotin to desthiobiotin in filtrates from cultures grown in medium containing citrulline, ornithine or lysine as the carbon and nitrogen source were approx. 1:1, 2:3 and $1: 2$ respectively.

The need for comparatively high concentrations of aspartate, citrulline, lysine or ornithine in the medium to obtain an appreciable stimulation in the production of compounds with biotin activity suggested that the effect was caused by products of metabolism of these amino acids rather than by incorporation of the intact amino acid into biotin and biotin-like compounds.

Catabolism of citrulline by bacteria leads to the production of carbamoyl phosphate and ornithine (Korzenovsky \& Werkman, 1953), and ornithine can undergo transamination to give glutamic semialdehyde (Meister, 1954). Glutamic semialdehyde is not amenable to testing for stimulation of production, but glutamate, which is probably formed from the semialdehyde, caused a comparatively small stimulation when included in the medium as the source of nitrogen or carbon and nitrogen (Table 3). Citrulline can also react with aspartate to give argininosuccinate, which in turn can give rise to arginine. Because of the high cost of the compound, the effect of argininosuccinate on the production of compounds with biotin activity was tested with smaller $(10 \mathrm{ml}$.) cultures. The addition of argininosuccinate to basal medium had no detectable effect
Table 4. Production of compounds with biotin activity by the pseudomonad in media containing arginine, argininosuccinate, citrulline or ornithine as the major nitrogen source

Compounds were included in media at $5 \mathrm{~mm}$. Cultures $(10 \mathrm{ml}$.$) were removed when growth reached the values$ indicated and the biotin activity in the culture filtrates was determined by the lactobacillus assay.

Biotin activity in filtrate ( $\mathrm{m} \mu \mathrm{g}$. of biotin equivalent/ml.)

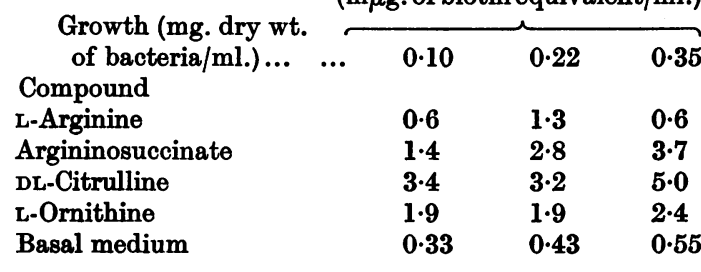

on production and the pseudomonad was unable to use this compound as a source of carbon and nitrogen. But when the compound was included in medium as the major source of nitrogen (at $5 \mathrm{~mm}$ ) there was an appreciable stimulation as compared with that caused by other amino acids (Table 4). Growth of the pseudomonad in medium containing argininosuccinate as the major source of nitrogen was extremely slow, about $200 \mathrm{hr}$. being required for the culture to reach a density of $0.35 \mathrm{mg}$. dry wt. of bacteria $/ \mathrm{ml}$. Chromatographic analysis of filtrates from cultures of the bacterium grown in this medium showed that only a small proportion of the amino acid was converted into the cyclized form (Walker \& Myers, 1953). Arginine, which is formed 
from argininosuccinate, was considerably less active than ornithine, citrulline or argininosuccinate (Tables 3 and 4). The bacteria did not grow in medium containing urea as the major source of carbon and nitrogen, and there was little if any stimulation in production in medium containing urea as the major nitrogen source.

Two pathways of lysine catabolism in bacteria have been reported. One of these proceeds via pipecolic acid and $\alpha$-aminoadipate (Schweet, Holden \& Lowy, 1954), whereas the other shorter pathway involves the production of 5-aminovalerate as an intermediate (Hagihara, Hayaishi, Ichihara \& Suda, 1960); both pathways result in the formation of glutarate. The only products of lysine catabolism that could be obtained for testing were glutarate and 5-aminovalerate. The former compound had no stimulatory effect on the production of compounds with biotin activity (Table 1), but 5-aminovalerate caused a little stimulation, though this was less than with lysine (Table 3). Fumarate and oxaloacetate, two possible products of the deamination of aspartate, had no detectable effect on production. Also, the stimulatory effect of asparagine was markedly less than that caused by aspartate.

\section{Production of compounds with biotin activity in media containing mixtures of stimulatory compounds}

An examination was made of the effect of certain mixtures of stimulatory compounds on the production of compounds with biotin activity with the aim of assessing the contribution that these compounds might make in biotin biosynthesis.

It seemed possible that malonate and pimelate might make similar biosynthetic contributions in view of the suggested participation of malonate in the biosynthesis of pimelate (Lezius et al. 1963). To test this hypothesis, the production of compounds with biotin activity was studied in media containing malonate or pimelate or a mixture of these acids as the carbon source. Because the lag phase of growth of the pseudomonad in medium containing pimelate as the carbon source was considerably longer than in media containing malonate as carbon source, the bacteria were grown in pimelate-containing medium and malonate, or additional pimelate or water was added aseptically to cultures as they entered the exponential phase of growth. The growth rate in the exponential phase was not markedly different in these media. The results in Table 5 show that the production of compounds with biotin activity in medium containing pimelate and malonate was no greater than in media containing malonate alone, which suggests that these compounds probably make a similar contribution in the biosynthesis of biotin and biotin-like compounds.

The effect of certain binary mixtures of stimulatory amino acids on the production of compounds with biotin activity was also examined. The addition of cysteine to medium containing citrulline, ornithine, arginine or lysine as the carbon and nitrogen source caused some stimulation, but the total production was never greater than that in the corresponding medium lacking cysteine.

Biotin activity in filtrates from cultures containing cysteine together with malonate as the carbon source and ammonium chloride as the nitrogen source was greater than the sum of the stimulations caused by malonate and cysteine individually (Fig. 3), which suggests that these compounds probably make different contributions in the biosynthesis of biotin and biotin-like compounds. In media containing malonate and citrulline, ornithine, lysine or aspartate the stimulation in production was equal to or slightly below the sum of the stimulations caused by malonate and the individual amino acid.

Certain heterocyclic compounds, including cytosine and thymine, were included in media containing malonate or individual amino acids as sources of

Table 5. Effect of malonate and pimelate, singly and together, on the production by the pseudomonad of compounds with biotin activity

The bacterium was grown in medium containing pimelate $(50 \mathrm{~mm})$ as the carbon source and, as the cultures $(50 \mathrm{ml}$.) entered the exponential phase of growth $(0.025 \mathrm{mg}$. dry wt. of bacteria $/ \mathrm{ml}$.; $72 \mathrm{hr}$.), additions of malonate $(10 \mathrm{ml}$.; final concn. $50 \mathrm{mM})$, pimelate $(10 \mathrm{ml}$.; final concn. $50 \mathrm{~mm})$ or water $(10 \mathrm{ml}$.) were made to the cultures. Portions of medium $(50 \mathrm{ml}$.) containing malonate $(50 \mathrm{~mm})$ were also inoculated at this time. Cultures were removed when growth reached $0.25 \mathrm{mg}$. dry wt. of bacteria $/ \mathrm{ml}$. and the total biotin activity of the culture filtrates was determined by the lactobacillus assay.

Biotin content of filtrate before additions were made

Carbon source ( $\mathrm{m} \mu \mathrm{g}$. of biotin equivalent/ml.) in medium

Pimelate

Pimelate

Pimelate

Malonate
$0 \cdot 18$

$0 \cdot 18$

$0 \cdot 18$

$0 \cdot 20$
Compound added to culture

Malonate

Pimelate

Water

None
Biotin content of filtrate

from culture containing

$0.25 \mathrm{mg}$. dry wt. $/ \mathrm{ml}$. (m $\mu$ g. of biotin equivalent/ml.)

$1 \cdot 26$

$0 \cdot 64$

0.60

$1 \cdot 24$ 


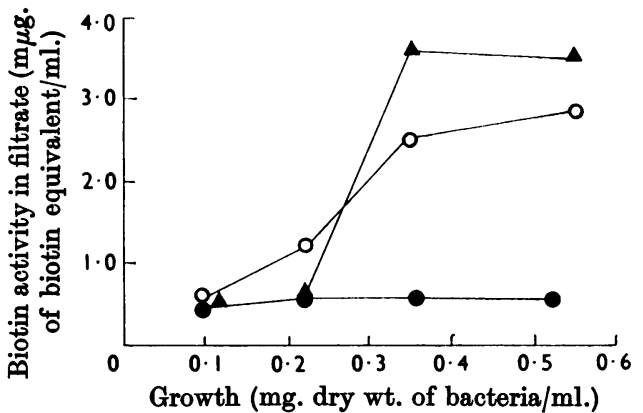

Fig. 3. Effect of cysteine or malonate on production of compounds with biotin activity. Bacteria were grown in basal medium (50 ml.) supplemented with cysteine (5 mM) (e) and in medium containing malonate as the carbon source $(50 \mathrm{~mm})$ either unsupplemented $(O)$ or containing cysteine $(5 \mathrm{~mm})(\Delta)$.

carbon, nitrogen or carbon and nitrogen, but none was found to stimulate the production of compounds with biotin activity.

\section{DISCUSSION}

The amounts of compounds with biotin activity produced by the pseudomonad used in the present study were considerably smaller than those produced by the strains of Phycomyces blakesleeanus (Schopfer, 1943; Eisenberg, 1963) and A. niger (Elford \& Wright, 1962) used in other studies. Nevertheless, the pseudomonad possesses certain advantages over these fungi, including a more rapid rate of growth and the ability to use a wider range of carbon and nitrogen sources. It resembles $P h$. blakesleeanus in producing desthiobiotin, biotin sulphoxide and certain unidentified biotin-like compounds in addition to biotin, and the relative proportions of biotin and desthiobiotin produced by the pseudomonad in basal medium were similar to those present in the mould culture filtrates (Eisenberg, 1963). Previous workers have experienced difficulty in measuring the relative proportions of biotin and desthiobiotin in filtrates because they were unable to separate these compounds chromatographically; this difficulty was overcome in the present study by using solvent system II. None of the unidentified biotin-like compounds produced by the pseudomonad appears to be identical with that synthesized by $P$ h. blakesleeanus, which, according to Eisenberg (1963), does not combine with avidin and is probably a monoamino acid.

The ability of pimelate to stimulate the production of compounds with biotin activity has been noted with other micro-organisms, and Eisenberg (1962) and Elford \& Wright (1962) have reported evidence for the incorporation of radioactive pimelate into the biotin molecule. But stimulation by malonate, which with the pseudomonad had a much greater effect than either pimelate or adipate, has not previously been reported. Since stimulation by malonate, adipate or pimelate occurred only when these acids were present in media as the major carbon source, it is likely that a product of metabolism of these acids rather than the unsubstituted compound is used in the biosynthesis of biotin and biotin-like compounds. The ability to utilize malonate is not widespread among micro-organisms, but it has been reported that a strain of Pseudomonas fuorescens can use this acid as a carbon source, and that the first reaction in the metabolism of malonate is the formation of malonyl-CoA (Wolf, Ivler \& Rittenberg, 1954; Hayaishi, 1955). The finding that malonate and pimelate probably make similar contributions in the biosynthesis of biotin and biotinlike compounds by the pseudomonad could lend support to the hypothesis of Lezius et al. (1963) that pimelyl-CoA may be formed from malonyl-CoA. The relatively greater production of biotin as compared with desthiobiotin in media containing malonate as the carbon source is difficult to explain. But, if it is assumed that malonate contributes carbon atoms mainly to the side chain of the biotin molecule, this finding could indicate that desthiobiotin is not an immediate precursor of biotin.

The ability of cysteine to stimulate the production of compounds with biotin activity, and particularly of biotin as compared with desthiobiotin, has been reported by Eakin \& Eakin (1942), although not in media lacking pimelate, and on general biochemical grounds it would be expected that the sulphur atom in the biotin molecule would be derived ultimately from the thiol group of cysteine. The inability of methionine to cause stimulation suggests that the $-\mathrm{CH}_{2}-\mathrm{S}-\mathrm{CH}_{2}-\mathrm{CH}_{2}-$ group, which could conceivably be contributed by this amino acid, is probably not used as such in biotin biosynthesis.

Much less is known about the origins of the carbon and nitrogen atoms in the ureido ring of the biotin molecule. But, in view of the inability of a number of heterocyclic compounds to stimulate the production of compounds with biotin activity by the pseudomonad, it is unlikely that the imidazole nucleus in the ureido ring is contributed intact. The incorporation of intact ring compounds during the biosynthesis of multicyclic molecules is comparatively uncommon, although it has been shown that the atoms in rings $B$ and $c$ of the riboflavine molecule can be supplied intact by purines (McNutt, 1954).

The stimulation caused by amino acids suggests that at least some of the atoms in the ureido ring arose from these compounds or from products of their metabolism. The slight stimulatory effect caused by amino acids when added individually to basal medium is probably due to their contributing 
to the pool of low-molecular-weight nitrogenous compounds in the bacterium. It is quite likely, too, that this explains the extra stimulation caused by lysine when this amino acid was included in the medium as the nitrogen or carbon and nitrogen source, since glutarate, which is an end product of lysine catabolism, had little stimulatory effect, whereas 5-aminovalerate, which is an intermediate on one of the pathways of lysine catabolism and contains one less amino group than lysine, had some stimulatory effect but much less than that of lysine. The much greater stimulation caused by ornithine, citrulline and argininosuccinate, when these amino acids were present individually in media as the carbon and nitrogen or nitrogen source, suggests that the ureido group in the biotin molecule might be derived from one or more of these amino acids or from products of their metabolism. Amino acid breakdown in Ps. fluorescens has been shown to be repressed by glucose (Jacoby, 1964), and the increased stimulation by amino acids when used as the carbon and nitrogen or nitrogen source could be due to the more extensive catabolism of the amino acid as compared with that in basal medium containing glucose. Since arginine had much less effect than ornithine, citrulline or argininosuccinate, it is possible that argininosuccinate or a product of metabolism of this amino acid is used directly in biotin biosynthesis. Further evidence for the role of these amino acids in furnishing the ureido group during biotin biosynthesis will have to be sought in experiments with radioactive labelled compounds, although these present considerable technical difficulties with the small amounts of biotin and biotinlike compounds produced by living cells.

This work was supported by grants for apparatus from the Medical Research Council and the North of England Committee of the British Empire Cancer Campaign. We also thank Miss Judith Hall for excellent technical assistance. M. I. thanks the Pakistan Council of Scientific and Industrial Research for a grant of study leave.

\section{REFERENCES}

Barton-Wright, E. C. (1952). The Microbiological Assay of the Vitamin B-Complex and Amino Acids, pp. 63-67. London: Pitman.
Campbell, L. L. \& Williams, O. B. (1953). J. Bact. 65, 146. du Vigneaud, V., Dittmer, K., Hague, E. \& Long, B. (1942a). Science, 96, 186.

du Vigneaud, V., Dittmer, K., Hofmann, K. \& Melville, D. B. (1942b). Proc. Soc. exp. Biol., N.Y., 50, 374.

Eakin, R. E. \& Eakin, E. A. (1942). Science, 96, 187.

Eakin, R. E., Snell, E. E. \& Williams, R. J. (1941). J. biol. Chem. 140, 535.

Eisenberg, M. A. (1962). Biochem. biophys. Res. Commun. $8,437$.

Eisenberg, M. A. (1963). J. Bact. 86, 673.

Elford, H. L. \& Wright, L. D. (1962). Fed. Proc. 21, 467.

Goodwin, T. W. (1963). Biosynthesis of Vitamins and Related Compounds, pp. 145-158. London: Academic Press (Inc.) Ltd.

Green, N. M. (1963). Biochem. J. 89, 599.

Hagihara, H., Hayaishi, H., Ichihara, A. \& Suda, M. (1960). J. Biochem., Tokyo, 48, 267.

Hayaishi, H. (1955). J. biol. Chem. 215, 125.

Hertz, R. (1943). Proc. Soc. exp. Biol., N.Y., 52, 15.

Hofmann, K., Melville, D. B. \& du Vigneaud, V. (1941). J. biol. Chem. 141, 207.

Jacoby, G. A. (1964). Biochem. J. 92, 1.

Korzenovsky, M. \& Werkman, C. H. (1953). Arch. Biochem. Biophys. 46, 174.

Landy, M. \& Dicken, D. M. (1941). Proc. Soc. exp. Biol., N.Y., 46, 449.

Leonian, L. H. \& Lilly, V. G. (1945). J. Bact. 49, 291.

Lezius, A., Ringelmann, E. \& Lynen, F. (1963). Biochem. Z. 336, 510.

Lichstein, H. C. (1955). Arch. Biochem. Biophys. 58, 423.

McNutt, W. S. (1954). J. biol. Chem. 210, 511.

Meister, A. (1954). J. biol. Chem. 206, 587.

Melville, D. B., Genghof, D. S. \& Lee, J. M. (1954). J. biol. Chem. 208, 503.

Northam, B. E. \& Norris, F. W. (1951). J. gen. Microbiol. $19,146$.

Rose, A. H. (1960). J. gen. Microbiol. 23, 143.

Rose, A. H. \& Nickerson, W. J. (1956). J. Bact. 72, 324.

Rose, A. H. \& Mahboob Ilahi (1963). Biochem. J. 89, 96P.

Schopfer, W. H. (1943). Z. Vitaminforsch. 14, 42.

Schweet, R. S., Holden, J. T. \& Lowy, P. H. (1954). J. biol. Chem. 211, 517.

Smith, A. M. \& Agiza, A. H. (1957). Analyst, 76, 623.

Tatum, E. L. (1945). J. biol. Chem. 160, 455.

Walker, J. B. \& Myers, J. (1953). J. biol. Chem. 203, 143.

Wolf, J. B., Ivler, D. \& Rittenberg, S. C. (1954). J. biol. Chem. 209, 867.

Wright, L. D., Cresson, E. L., Valiant, J., Wolf, D. E. \& Folkers, K. (1954). J. Amer. chem. Soc. 76, 4163.

Wright, L. D. \& Driscoll, C. A. (1954). J. Amer. chem. Soc. 76, 4999. 